\title{
Power Line Carrier Communications and its Interest in the Current Power Grid Scenario
}

\author{
O.Abarrategui ${ }^{1}$, I. Zamora ${ }^{2}$, DM. Larruskain ${ }^{3}$ and A. Iturregi ${ }^{4}$ \\ ${ }^{1}$ Department of Electrical Engineering \\ UPV-EHU \\ Colina Beurko s/n, 48901 Barakaldo (Spain) \\ Phone/Fax number:+0034 94601 1971, e-mail: oihane.abarrategui@ehu.es ${ }^{1}$, inmaculada.zamora@ehu.es $^{2}$, \\ marene.larruskain@ehu.es $^{3}$, araitz.iturregi@ehu.es $^{4}$
}

\begin{abstract}
During the last years PLC technologies have been widely developed mainly due to new modulation techniques used for wireless telecommunication systems that can also be applied to PLC systems. The current state of the art of PLC communications is presents many possibilities and opportunities for the utilities.
\end{abstract}

Besides the Distributed Energy Resources integration to the distribution grid is

The aim of this paper is to make a review of the existing PLC technologies as well as the analysis of an interesting range of electric applications of PLC.

\section{Key words}

Power Line Carrier (PLC), Smart Grid, Active Network, Distributed Energy Resources (DER).

\section{Introduction}

Power Line Carrier communication systems consist of a high frequency signal injection over the electrical power lines. This kind of technology has been used since the 1950 decade in order to provide signalling and ripple control in High Voltage lines, at transmission level.

In the last years the interest for this technology has suffered a revival because the impressing increase of the mobile telecommunications has brought a big development in transmission technologies for this kind of communications. In particular, new modulation technologies used for wireless communication are especially suitable for PLC communication and make massive data transmissions possible.

Besides, the opening of the market, the need to integrate Distributed Energy Resources (DER) and the increase of the power supply demand create a new scenario in which the approach of the energy distribution system has to change.
In such a scenario, the distribution system needs to be automated in order to give a satisfactory response to the problems that will eventually appear. The actual automation ratio of the Spanish distribution system is of approximately a $2 \%$ or $3 \%$ and the prevision is that it should increase to more than a $50 \%$ in the following years.

Currently PLC communications can be broadband as well as narrowband and both cases present successful transmissions.

In the case of Narrowband PLC there are the CENELEC standards EN50065 and EN50065-1, for signals between $3 \mathrm{kHz}$ and $148 \mathrm{kHz}$ over low voltage public and/or private grids. For Broadband PLC, European Commission funded OPERA IST and Opera 2 projects are working towards the standard development currently. In this case the used signals range from $1 \mathrm{MHz}$ to $34 \mathrm{MHz}$ with a bandwidth between 10 and $30 \mathrm{MHz}$ and a bit rate of $200 \mathrm{mbps}$. The results of the both above mentioned projects will feed the ETSI Broadband Powerline Telecommunications standard.

Thus, it would be possible to think of an automated distribution scenario with PLC used as a communication link used for multiple applications.

\section{PLC Network: Structure and Topology}

PLC networks have a parallel structure to the electricity network and thus show several similarities with it. In this point an analysis of the typical PLC structure and the usual topologies of such a grid will be done.

\section{A. Structure}

A PLC network is divided in two main parts. On the one hand there will be a PLC network parallel to the medium voltage grid and on the other a PLC network parallel to the low voltage grid. These networks are named MV PLC and LV PLC networks respectively. The boundary and end components of the network are shown in Fig 2. 


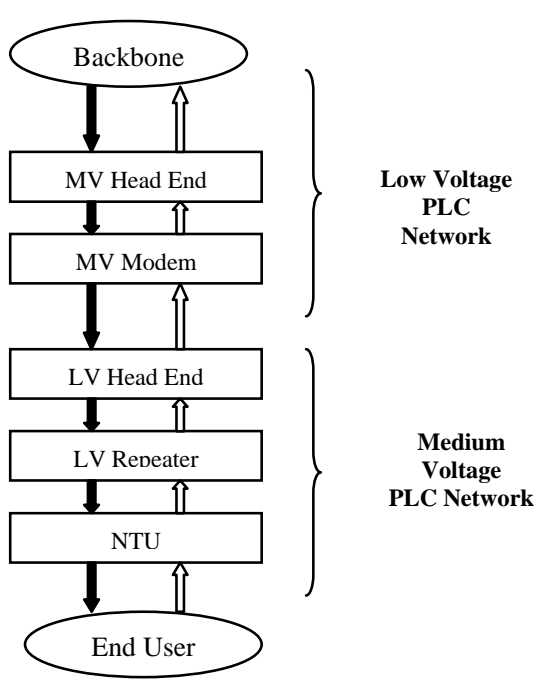

Fig. 2. PLC Network Structure

Medium Voltage Head End: It enables the communication between the Backbone or the main communications network and the PLC network.

Medium Voltage Modem: it is the interface between a Medium Voltage PLC Network and a Low Voltage PLC network on the Medium Voltage side.

Low Voltage Head End: It represents the end of the Low Voltage PLC network and is a gateway to the Medium Voltage network which can be PLC or otherwise. It is normally placed on the distribution transformer which acts as a natural low pass filter for the high frequency signal injected in the network.

Low Voltage Repeater: In case of lines of significant distances between the Head End and the Network Termination Unit it will be necessary to place Repeater Units along the line in order not to lose the high frequency signal.

Network Termination Unit (NTU): It is the interface between the client equipment and the low voltage PLC network. It is normally placed at the client premises.

\section{B. Topology}

The PLC Network topology depends strongly on the distribution grid topology and is similar to it. Thus, it can be meshed or radial and generally the MV Head and LV Head end are placed at substation and transformation centre levels respectively.

In MV PLC netoworks, there are two main parameters that directly affect the network topology: distance and latency. These two parameters determine the kind of repeaters that are normally used on PLC networks.

Time Division (TD) Repeaters: If the link between the End User and Head End is made using the whole frequency band (generally 16 to $30 \mathrm{MHz}$ ), then the link between repeater and Head End will have to be made using the same frequency band but during a different time slot. The TD Repeaters design is simple but the latency is big and the bandwidth management is not especially effective.

Frequency Division Repeaters: if the link between End User and Head End only uses a part of the frequency band, then the link between the repeater and the Head End is able to use another frequency for its transmission. Thus, it is possible to send two different signals within the same frequency band. The latency is low and the use frequencies much more efficient than in TD repeaters. However, the design is more complicated and the prices higher.

Fig.3 shows a MV network topology using both TD and FD Repeaters:

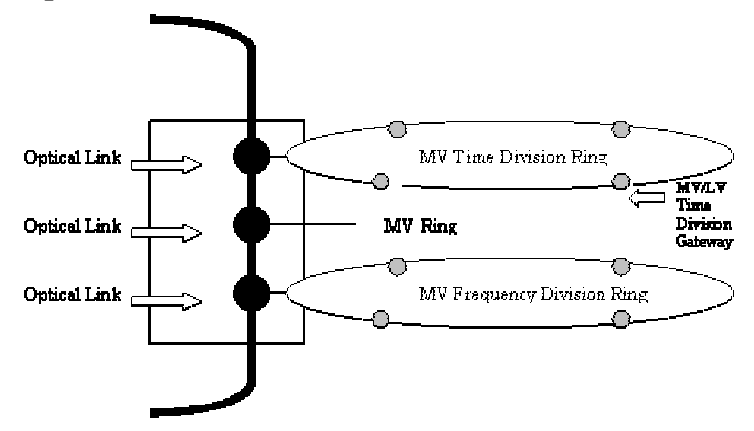

Fig. 3. MV PLC Network Topology

There are different LV PLC network topologies depending on the area which can be rural, residential or urban, among others. For an urban area with high apartment and office buildings and a centralized counter room a typical LV PLC topology would be the one shown in Fig.4.
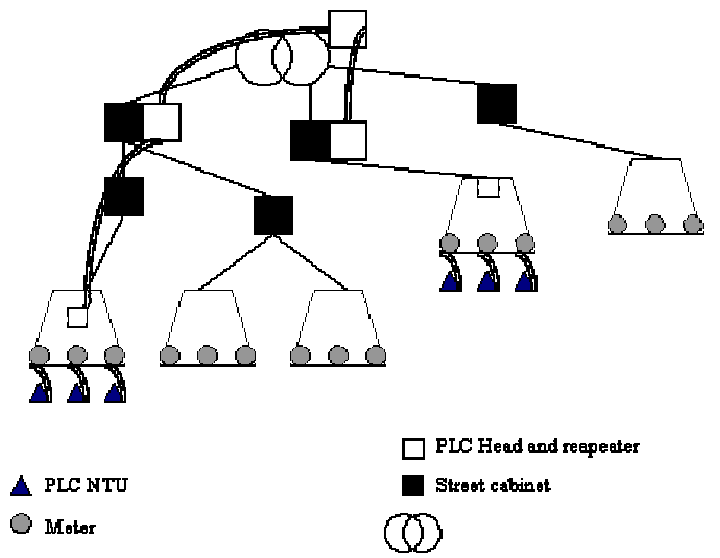

Fig.4. LV PLC Network Topology

\section{PLC Modulation and Transmission}

\section{A.Modulation}

Modulation techniques have made a big progress during the last decade, due to the big developments in mobile communications. These developments make possible for 
PLC networks to be more resilient against Electromagnetic Interferences.

Thus, the bandwidth and the speed of PLC communications have allowed a more complex and faster data flow and now is possible to expand the range of applications for this kind of networks.

The modulations used in PLC networks are the following:

- Orthogonal Frequency Division Multiplexing (OFDM): This modulation is based upon the orthogonality of the carriers. The carriers are chosen so that they are orthogonal among themselves. This minimizes the crosstalk and contributes to elevate the spectral efficiency which is close to the Nyquist rate. Thus, it is possible to use almost the whole frequency range. Besides, OFDM has a white spectrum due to the favourable electromagnetic properties. OFDM modulation requires a very precise synchronization between the transmitter and the receiver, because any deviation of the sub-carriers would affect the orthogonality. This modulation technique is used by DS2.

- $\quad$ Gaussian Minimum Shift Keying (GMSK): This kind of modulation is an evolution of the FSK modulation. The bandwidth of the signal changes depending in the binary digit that has to be transmitted and the bandwidth can be controlled by a low-pass Gaussian filter. GMSK is the modulation used by ASCOM.

- $\quad$ Direct Sequence Spread Spectrum(DSSS): This kind of modulation is also known as DSCDMA, Direct Sequence Code Division Multiple Access and it has been defined by the IEEE in the standard 802.11 for local wireless networks. DSSS uses a pseudo-noise code to modulate the carrier, thus increasing the bandwidth of the transmission and decreasing the spectral density power. The resulting signal's spectrum is similar to the noise frequency spectrum, so every receptor considers it noise except the one the signal is being directed to. DSSS was the modulation technique used by Main.net.

DS2 has been chosen by Opera so very probably OFDM will be the European standard. This three modulation techniques are noise resilient and make satisfactory bandwidth management.

\section{B. Transmission Problems}

The parameters that affect the PLC transmission and thus have to be considered are: attenuation, noise, noise to signal relation, crosstalk and delay spread.
A.Attenuation: Attenuation is the loss of power that a high frequency signal suffers through the transmission. To measure attenuation, two locations have to be considered: outside the buildings (generally between the transformation centre and the point of access to the building) and the other one inside the building, generally from the general distribution point or central counters to the end customer socket.

Attenuation is a function of the frequency and the distance, so for the same network the higher the information carrier is, the bigger the attenuation. Besides, the bigger the transmission line is the bigger the attenuation. Table I shows the parameters that define the attenuation:

Table I

\begin{tabular}{|c|c|c|}
\hline Parameter & Units & Meaning \\
\hline c & $\mathrm{dB} / \mathbf{m} / \mathbf{M H z}$ & $\begin{array}{l}\text { Frequency and distance dependent } \\
\text { attenuation }\end{array}$ \\
\hline $\mathbf{d}$ & $\mathrm{dB} / \mathrm{MHz}$ & Frequency dependent attenuation \\
\hline$\overline{\mathbf{e}}$ & $\mathrm{dB} / \mathbf{m}$ & Distance dependent attenuation \\
\hline $\mathbf{g}$ & dB & Base attenuation \\
\hline
\end{tabular}

Thus, the final attenuation can be calculated through the following equation:

$\mu_{A}(f, D)=c \cdot f \cdot D+d \cdot f+e \cdot D+g$

B.Noise: The noise signal is generated by interferences and occupies part of the frequency spectrum. The short wave transmissions create noise and may affect the PLC communications. In PLC networks the noise signal has to be measured in different positions of the network. Generally, measurements are made at transformation centres or substations, for LV PLC and MV PLC networks respectively, central meters or at the customer socket, depending if the measurements are going to be outdoor or indoor. This are considered the most significant positions of the network.

Unlike the attenuation, the noise threshold diminishes as the carrier signal frequency increases.

For certain predictable interferences that cause noise in a determined frequency, notch filters can be used with good results.

C. Signal-to-Noise relation SNR: This parameter gives information about how the noise signal affects the information carrier signal, as it is a relation between the carrier signal in $\mathrm{dB}$ and the noise signal in $\mathrm{dB}$ at a certain point. It can be calculated knowing the attenuation, noise and power of the transmitted signal:

$P_{t x}-A=P_{r x}$

$S N R=P_{r x}-N_{r x}=P_{t x}-A-N_{r x}$

Where, $\mathrm{P}_{\mathrm{tx}}$ is the power of the transmitted signal, A the attenuation $\mathrm{N}_{\mathrm{rx}}$ the noise at the receiver and $\mathrm{P}_{\mathrm{rx}}$ the power of the signal at the receiver.

D. Crosstalk: It defines the isolation between a MV PLC network and a low voltage PLC network. The crosstalk measurements are done on both sides of the transformer, for each voltage side. It gives information about how both PLC networks interfere with each other. 
As other attenuation measurements, crosstalk attenuation depends on frequency, so the PLC MV and LV networks will interfere less with each other:

$$
A=a f^{3}+b f^{2}+c f+d
$$

Where, $\mathrm{A}$ is the crosstalk attenuation, $f$ the frequency in $\mathrm{MHz}$ and $\mathrm{a}, \mathrm{b}, \mathrm{c}$ and $\mathrm{d}$ the adjustment coefficients shown in Table II.

Table II

\begin{tabular}{|l|l|l|}
\hline Coefficient & Average couplings & Minimum couplings \\
\hline a & 0,00077 & 0,00073 \\
\hline B & $-0,0817$ & $-0,0835$ \\
\hline C & 2,258 & 2,545 \\
\hline D & 54,22 & 33,68 \\
\hline
\end{tabular}

E. Spread Delay: The PLC networks present a much branched transmission mean. This causes important signal reflexion. The paths of the reflected signals have different distances and because of this, the reflected signals arrive in the receiver at different times. The time difference between the first signal and the last of the reflexions that reaches the receptor is called Spread Delay or Delay Spread.

$$
D S=\sqrt{\frac{\int_{0}^{t_{1}}\left[h(t)^{2} t^{2}\right] d t}{\int_{0}^{t_{1}} h(t)^{2} d t}}
$$

\section{PLC applications and interest}

As we mentioned in the introduction, the interest of PLC communications is growing, due to the current circumstances. The active grid or intelligence grid concept will define the network of the future and needs of a parallel communication system in order to give a successful response to its problems.

A. Industrial Automation: In an industrial environment the PLC communication networks can be used to give electric energy related services, such as meter reading, demand management and remote billing but also to give value added services like remote control and security, automation or even, education, information and ebusiness opportunities. On the other hand it can also offer telecommunication services such as traditional telephony and Internet.

The cost of a PLC network for a medium size industry is shown in Table III:

\begin{tabular}{|l|c|c|c|c|}
\hline & $\begin{array}{l}\text { Tead End } \\
\text { Modem } \\
\text { (transformer) }\end{array}$ & Gateway & $\begin{array}{l}\text { PLC } \\
\text { Modem } \\
\text { (client } \\
\text { premises) }\end{array}$ & $\begin{array}{l}\text { Total } \\
\text { Cost(€) }\end{array}$ \\
\hline $\begin{array}{l}\text { Number } \\
\text { of units }\end{array}$ & 1 & 20 & 220 & \\
\hline $\begin{array}{l}\text { Cost per } \\
\text { unit(€) }\end{array}$ & 500 & 100 & 100 & 24500 \\
\hline $\begin{array}{l}\text { Total } \\
\text { cost(€) }\end{array}$ & 500 & 2000 & 22000 & \\
\hline
\end{tabular}

B. Telecommunication services: Current PLC networks are able to reach speeds of $200 \mathrm{Mbps}$. Telephony and Internet services can be delivered at high speed through broadband PLC networks. Traditional telephony uses Plesiochronous Digital Hierarchy, PDH. PDH uses Time Division Multiplexing, TDM. One possibility is to send the TDM frame over IP, and the voice over TDM, VoTDM. However, this service should accomplish the quality and reliability criteria, like Bit Error Rate, timing and latency, and unfortunately the delay in VoTDM transmissions exceeds $25 \mathrm{~ms}$. Nevertheless, it is possible to give a good telephony service over IP. Over TCP/IP, VoIP and Internet services can be delivered at a 200Mbps speed, so it can be possible to compete with technologies such as ADSL.

\section{Solutions for electricity suppliers}

c.1. Ripple Control: It is used for applications such as switched meters, local lightning, connection and disconnection of load groups, generation units and individual controls. Traditionally, it has been done through a radio communication through an antenna covering a $500 \mathrm{~km}$ range, which allows only a unidirectional communication. PLC would allow a bidirectional communication and makes communications more flexible and allows different applications.

c.2. Transformation centre's telecontrol: Transformation centre's operation and control functions could be done via PLC. Currently, the distribution networks automation is of $2 \%$ but in the near future it is expected to reach $50 \%$.

c.3 Fraud detection: The energy provided by the transformer and the energy reaching the end customer could be compared, losses more precisely calculated and fraud detected.

c.4. Demand Side Management: Demand side management systems cover a variety of policies in order to decrease the customer's energy consumption, increase the energy efficiency, reduce the energy costs, vary the energy use times and promote different energy sources. Instead of building new power plants, consumption could be reduced by implementing special programs for particular clients, industries or institutions.

c.5. Distributed Generation integration: The distributed generation will grow significantly during the next years. In thirty years the amount of energy produce by distributed generation units will equal to the conventionally produced one. To minimize the impact on the stability and functioning of the grid, the communication between installations and equipment will have to be guaranteed. For this purpose, PLC is a feasible technology.

c.6. System protection: The communication link can be used to transit control signals that may be used to protect the system. For example, PLC can be successfully used in order to detect islanding operation of DER units.

c.7. Fault detection: The synergies between the PLC communication system could be analyzed and used for fault detection and location and also to do preventive maintenance actions that can improve significantly the grid operation. 


\section{Conclusion}

The electric power grid is about to face an important renewal in which the information and communication technologies are of vital importance. Thus, it is important to have a solid communication infrastructure. Currently, PLC networks provide a proprietary solution and enough reliability and quality conditions.

When designing or analyzing a PLC network, the structure and components of it, as well as its topology have to be taken into account. Also, there are different providers that use different technologies so compatibility is an issue. There is a narrowband standard and the broadband is very developed.

The transmission quality is also an important factor. In order to characterize a PLC network, attenuation, noise, SNR, crosstalk and Delay Spread parameters have to be measured, analyzed and considered.

Finally, the application range of PLC is very wide, it can provide telecommunication services and value added services but it can also be very useful for distribution and transmission system operators in order to guarantee the operation and control of the power grid.

\section{References}

[1] The OPERA IST Integrated Project No 507667 funded by EC Public Deliverables. www.ist-opera.org

[2] J.G. Proakis "Digital Communication". Edition McGraw-Hill Inc. 2001

[3] Elisa Garcia, Miguel A. Chileno, Luis Legorburu "The OPERA IST Project", 9 CHLIE Marbella, Spain, July 2005.

[4] O. Abarrategui, I. Zamora, DM. .Larruskain, M. Gomez "DLC Communications for Islanding Detection in Systems with DG" XCLEE Funchal, Madeira, Portugal, Spain, July 2007.

[5] O. Abarrategui, I. Zamora, DM. Larruskain, M. Gomez "Smart Grid: A Global Vision" XCLEE Funchal, Madeira, Portugal, Spain, July 2007.

[6] Elisa Garcia, Miguel A. Chileno, Luis Legorburu "The experience of the Iberdrola Group in Power Line Communications", 9 CHLIE Marbella, Spain, July 2005. 